\title{
Damage localization method for plates based on the time reversal of the mode-converted Lamb waves
}

$\operatorname{AUTHOR}(\mathrm{S})$ :

Mori, Naoki; Biwa, Shiro; Kusaka, Takayuki

\section{CITATION:}

Mori, Naoki ...[et al]. Damage localization method for plates based on the time reversal of the mode-converted Lamb waves. Ultrasonics 2019, 91: $19-29$

ISSUE DATE:

2019-01

URL:

http://hdl.handle.net/2433/236056

\section{RIGHT:}

(c) <2019>. This manuscript version is made available under the CC-BY-NC-ND 4.0 license

http://creativecommons.org/licenses/by-nc-nd/4.0/.; The full-text file will be made open to the public on 01 January 2021 in accordance with publisher's 'Terms and Conditions for Self-Archiving'.; This is not the published version. Please cite only the published version,; この論文は出版社版でありません。引用の際には出版社版をご確認ご利用ください。 


\title{
Damage localization method for plates based on the time reversal of the mode-converted Lamb waves
}

\author{
Naoki Mori $^{\mathrm{a}, *}$, Shiro Biwa ${ }^{\mathrm{b}}$, Takayuki Kusaka ${ }^{\mathrm{a}}$ \\ ${ }^{a}$ Department of Mechanical Engineering, College of Science and Engineering, \\ Ritsumeikan University, 1-1-1 Noji-higashi, Kusatsu, Shiga 525-8577, Japan. \\ ${ }^{\mathrm{b}}$ Department of Aeronautics and Astronautics, Graduate School of Engineering, \\ Kyoto University, Katsura, Nishikyo-ku, Kyoto 615-8540, Japan. \\ ${ }^{*}$ Corresponding author. Tel: $+81-77-561-4838$. \\ E-mail address: nmori@fc.ritsumei.ac.jp (N. Mori).
}

\begin{abstract}
A damage localization method based on the time reversal focusing of the mode-converted scattered Lamb wave is proposed for plate structures with a non-symmetric defect in the thickness direction. Dual transducers are attached symmetrically on the upper and lower surfaces of the plate to selectively emit and receive the lowest-order symmetric (S0) and antisymmetric (A0) modes. The localization of damage is achieved by the numerical time-reversed (TR) simulation of the mode-converted Lamb wave generated at the defect. To investigate the validity of the proposed method, the signals of the Lamb waves in a plate with a partial-thickness notch are numerically simulated by the three-dimensional elastodynamic finite integration technique (EFIT). When the S0 mode is emitted in the damaged plate, not only the S0 mode is scattered but also the A0 mode is generated due to mode conversion at the notch. Similar mode conversion behavior is confirmed when the A0 mode is emitted. The time reversal of the mode-converted scattered Lamb waves creates focused spots at the damage location without using baseline data for the undamaged plate. The proposed method reduces the magnitude of the artifacts compared to the time reversal of the non-mode-converted Lamb wave, and yields the focused spot whose size is associated with the size of the notch and the half wavelength of the time-reversed wave mode. Furthermore, the proposed method is applied to a plate with a notch-type defect adjacent to an a priori known through-thickness hole, demonstrating the damage localization in a relatively complicated structure.
\end{abstract}

Keywords: Damage localization; Time reversal; Lamb wave; Mode conversion 


\section{Introduction}

Time reversal in acoustics is a wave focusing technique used in various areas, e.g. nondestructive evaluation, medical diagnosis, seismology, etc. When the wave signals recorded by multiple transducers are time-reversed and sent back into the medium, the self-focusing of the wave occurs at its original wave source. Since Fink and co-workers [1-3] established the concept of a time reversal mirror, time reversal acoustics has been applied not only to the focusing of acoustic waves at a desired location and time [4-6] but also to the localization of unknown wave sources or scatterers in various media [7-16].

Lamb waves offer an effective tool for the damage detection and localization in plate-like structures since they can propagate relatively long distances. However, as the propagation distance becomes longer, the time resolution of Lamb wave signals tends to be lower due to their dispersion and multi-modal characteristics. To overcome this difficulty, signal processing techniques such as dispersion compensation [17], two-dimensional Fourier transform [18], and wavelet transform [19-21] were proposed in foregoing studies. These approaches effectively work to determine the arrival time of the dispersive wave modes or to decompose the measured waveform into different modes, but require a priori knowledge about the dispersion and multi-modal characteristics.

Compensation of the Lamb wave dispersion characteristics can be achieved without a priori knowledge through the time reversal process, as reported by Ing and Fink [22]. They developed a time reversal mirror of a 32-element transducer array and demonstrated the self-compensation of the Lamb wave dispersion property using this device. In recent years, the temporal focusing of Lamb waves gathers attention in the damage detection for plate-like structures [23-28]. Park et al. [26] analyzed the time reversal process of the lowest-order antisymmetric (A0) mode which was emitted and received in pitch-catch mode. Their theoretical investigation has shown that the dispersion effect of the A0 mode is compensated in the backward propagation. It has been also shown that the main mode signal (i.e. the temporally focused signal obtained via the time reversal process) is accompanied by several sidebands due to the multi-modal effect of Lamb waves. The presence of possible damage can be evaluated from a damage index which is based on the difference between the input and reconstructed signals in the time reversal process $[25,27]$. Basically, only the damage located between the emitter and the receiver can be directly detected by this method, although the reconstruction algorithm for the probabilistic inspection of damage (RAPID) was incorporated into the time reversal technique to overcome this difficulty [28].

Spatial focusing of the time-reversed Lamb waves is another approach for the damage localization in plate structures. For a homogeneous plate, several studies have reported the spatial characteristics of the Lamb wave focusing through the time reversal process [29-31]. Park [30] demonstrated theoretically and numerically that the spatial resolution of a focused spot obtained by the time-reversed A0 mode in a homogeneous plate is closely associated with its half wavelength. For the damage localization in plate structures, Wang et al. [32] proposed a synthetic time-reversal method, which incorporated the time reversal concept into the synthetic aperture 
method. This approach was applied to estimate the location of a circular disk-shaped mass bonded on an aluminum alloy plate. Their concept was extended by Yu and Leckey [33] who evaluated the location and size of an electrical discharge machined slit by the multiplication of the time-reversed signals measured at multiple sensors. In the damage localization by the spatial focusing proposed in previous studies [32-34], however, baseline data for an undamaged structure are usually required to obtain the signals of the scattered wave from the damage because the measured waveforms include the direct wave from an actuator. Furthermore, the time reversal process of Lamb waves is treated merely as time shifting, which is applicable only to non-dispersive Lamb modes.

The aim of this study is to propose a time reversal method of Lamb waves for the damage localization in plates without using baseline data. To this purpose, the mode conversion induced by the damage is incorporated in the time reversal process. Dual transducers are attached symmetrically on both surfaces of the plate to selectively emit and receive the lowest-order symmetric (S0) and antisymmetric (A0) modes in a low frequency range. The damage localization is performed by the numerical time-reversed (TR) simulation of the mode-converted scattered Lamb wave.

In this study, to validate the proposed method, the signals of the Lamb wave in a plate with a partial-thickness notch-type defect are numerically simulated by the elastodynamic finite integration technique (EFIT) [35] instead of the actual measurement. The proposed damage localization method is presented in Section 2, and its verification procedure by the numerical simulation is explained in Section 3. Prior to the discussion of the time reversal of Lamb waves in the damaged plate, the effect of mode types and frequency on the focusing characteristics is studied for a homogeneous plate with no damage in Section 4. Subsequently, the time reversal of the scattered S0 and A0 modes at the notch is examined in Sections 5 and 6.

\section{Proposal of the damage localization method based on the time reversal of the mode-converted Lamb wave}

\subsection{Measurement of Lamb wave signals using dual transducers}

A homogeneous and isotropic elastic plate of uniform thickness $|z|<d / 2$ is considered in the $x-y-z$ Cartesian coordinate system, where $d$ is the plate thickness. In such a structure, Lamb waves and shear horizontal $(\mathrm{SH})$ guided waves can propagate along the plate. Lamb waves can be classified into symmetric and antisymmetric modes, which can be selectively excited and detected by dual transducers mounted symmetrically on the upper and lower surfaces of the structure $[36,37]$. The polarization direction of the transducers is assumed to be aligned in the thickness $(z)$ direction. In a sufficiently low frequency range, the propagating modes of the Lamb wave in the plate are only the $\mathrm{S} 0$ and $\mathrm{A} 0$ modes.

The aim of the proposed method is the localization of a defect with non-symmetric geometry in the thickness direction, without using baseline data. This type of defect is common and can be made by corrosion pitting, for example. As shown in Fig. 1, the proposed method consists of the 
following two steps:

(a) Emitting the S0 or A0 mode in a damaged plate, receiving the wave signals at multiple transducers mounted on the plate, and extracting the signals of the mode-converted scattered Lamb wave from the defect

(b) Localizing the damage by simulating the time reversal focusing of the mode-converted scattered wave

When the S0 mode is excited by a dual actuator in a frequency range below the cut-off frequencies of the higher-order guided modes, not only the S0 mode is scattered but also the A0 mode and the lowest-order shear horizontal ( $\mathrm{SH} 0$ ) mode are generated by mode conversion at the defect. In this situation, the electric voltage measured at the upper side of a dual transducer (Transducer $i$ ) is given as

$$
e_{i}^{\mathrm{U}}(t)=e_{\mathrm{S}, i}^{\mathrm{Dir}}(t)+e_{\mathrm{S}, i}^{\mathrm{Sca}}(t)+e_{\mathrm{A}, i}^{\mathrm{Sca}}(t)
$$

where $t$ is time, $e_{\mathrm{S}, i}^{\mathrm{Dir}}(t)$ is the signal of the direct $\mathrm{S} 0$ mode from the actuator, $e_{\mathrm{S}, i}^{\mathrm{Sca}}(t)$ and $e_{\mathrm{A}, i}^{\mathrm{Sca}}(t)$ are the signals of the scattered $\mathrm{S} 0$ and A0 modes from the defect, respectively $(i=1,2, \ldots$,

(a) Measurement of the mode-converted Lamb wave (e.g. Excitation of the S0 mode)
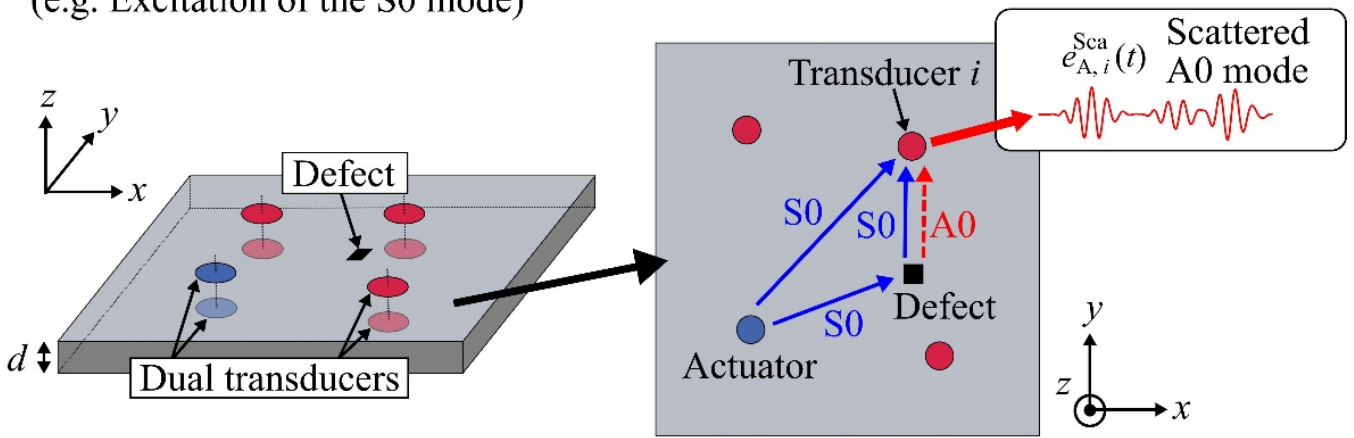

(b) Damage localization by numerical time-reversed (TR) simulation (Time reversal of the A0 mode)

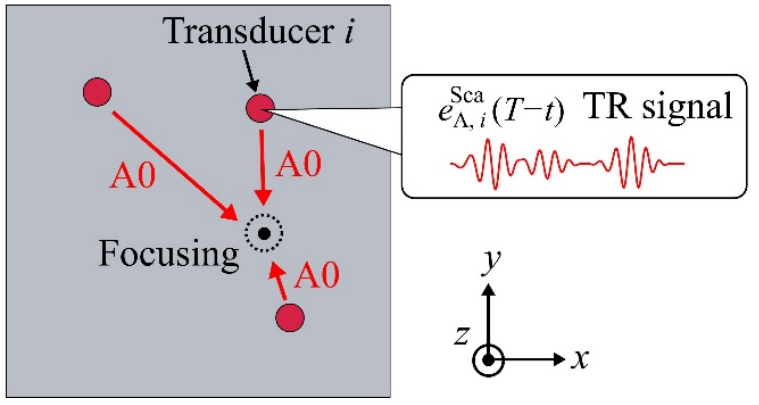

Fig. 1 Schematics of the proposed damage localization method: (a) the Lamb wave measurement using dual transducers and (b) the numerical time-reversed (TR) simulation of the mode-converted scattered Lamb wave at the defect. 
$N_{\mathrm{T}}$ ). The in-plane vibration of the $\mathrm{SH} 0$ mode is not detected by these transducers. The received signal at the other side of Transducer $i$ can be expressed as

$$
e_{i}^{\mathrm{L}}(t)=e_{\mathrm{S}, i}^{\mathrm{Dir}}(t)+e_{\mathrm{S}, i}^{\mathrm{Sca}}(t)-e_{\mathrm{A}, i}^{\mathrm{Sca}}(t)
$$

due to the symmetry of Lamb modes. The waveforms of the S0 and A0 modes, $e_{\mathrm{S}, i}^{\mathrm{Dir}}(t)+$ $e_{\mathrm{S}, i}^{\mathrm{Sca}}(t)$ and $e_{\mathrm{A}, i}^{\mathrm{Sca}}(t)$, can be isolated from the summation and subtraction of the signals $e_{i}^{\mathrm{U}}(t)$ and $e_{i}^{\mathrm{L}}(t)$, respectively. The signal of the S0 mode includes both the direct wave from the actuator and the scattered wave from the defect, but the waveform of the scattered A0 mode can be directly obtained from $e_{i}^{\mathrm{U}}(t)$ and $e_{i}^{\mathrm{L}}(t)$. When the A0 mode is emitted in a damaged plate, the scattered $\mathrm{S} 0$ mode from the defect can be directly calculated in a similar manner.

\subsection{Damage localization by numerical time-reversed (TR) simulation}

Based on the measured signals of the mode-converted Lamb wave generated at the defect, damage localization is performed by numerical TR simulation for the undamaged structure whose geometry and elastic property are assumed to be known. The propagation of the time-reversed Lamb wave is analyzed based on Hooke's law and the equations of motion in the three-dimensional elastodynamic theory. When the S0 mode is emitted in the damaged plate, the time-reversed signal of the scattered A 0 mode

$$
e_{i}^{\mathrm{R}}(t)=e_{\mathrm{A}, i}^{\mathrm{Sca}}(T-t),
$$

is used as the input waveform into Transducer $i$, and the A0 mode is re-emitted in the numerical TR simulation $(T>0)$, as shown in Fig. 1(b). Normal tractions $T_{z, i}^{\mathrm{U}}(t)$ and $T_{z, i}^{\mathrm{L}}(t)$ are prescribed uniformly in the regions on the upper and lower surfaces of the plate corresponding to Transducer $i$, respectively, where

$$
T_{z, i}^{\mathrm{U}}(t)=-T_{z, i}^{\mathrm{L}}(t)=k_{\mathrm{a}} e_{i}^{\mathrm{R}}(t)=k_{\mathrm{a}} e_{\mathrm{A}, i}^{\mathrm{Sca}}(T-t),
$$

and $k_{\mathrm{a}}$ represents the electro-mechanical efficiency coefficient of the transducer. In this study, the frequency dependence of the electro-mechanical efficiency coefficient is not taken into account because a narrow frequency range is used. On the other hand, when the A0 mode is emitted in the damaged plate, normal tractions are prescribed as

$$
T_{z, i}^{\mathrm{U}}(t)=T_{z, i}^{\mathrm{L}}(t)=k_{\mathrm{a}} e_{i}^{\mathrm{R}}(t)=k_{\mathrm{a}} e_{\mathrm{S}, i}^{\mathrm{Sca}}(T-t),
$$

at Transducer $i$ to re-emit the time-reversed S0 mode in the numerical TR simulation.

The locations of the focusing peaks are identified using the maximum absolute value of the out-of-plane particle velocity $v_{z}(x, y, z, t)$ on the plate surface

$$
I_{\mathrm{m}}(x, y)=\max _{0<t<T}\left|v_{z}(x, y, d / 2, t)\right| / v_{0}
$$


where $v_{0}$ is a normalization factor. In this study, the quantity $I_{\mathrm{m}}(x, y)$ is called focusing index, which is similar to the directivity pattern defined in Ref. [30]. Since the mode-converted Lamb wave originates from the damage, the focusing peak so obtained indicates the damage location in the plate.

\section{Verification of the proposed damage localization method}

In what follows, the time reversal process proposed in Section 2 is applied to the numerical model of a plate with a partial-thickness notch-type defect in order to investigate the validity of the proposed method. The signals of the forward-propagating Lamb waves are obtained by numerical simulation instead of the actual measurement. As shown in Fig. 2, the dimensions of the plate are $600 \mathrm{~mm}$ ( $x$ direction $) \times 600 \mathrm{~mm}$ ( $y$ direction), and the plate thickness is $d=5 \mathrm{~mm}$. The upper and lower surfaces $(|z|=d / 2)$ and the edge faces (e.g. $x=0)$ of the plate are assumed to be traction-free. The partial-thickness notch has a square shape (side lengths $10 \mathrm{~mm}$ in the $x$ and $y$ directions), and its depth in the $z$ direction is $d / 2=2.5 \mathrm{~mm}$.

\subsection{Numerical simulation of the forward-propagating SO and AO modes}

As described in Section 2.1, a dual actuator is attached symmetrically on the upper and lower surfaces of the plate to selectively emit the S0 or A0 mode in a low frequency range. The excitation of the Lamb waves is modeled analogously to the numerical TR simulation described in Section 2.2. Namely, the normal traction $T_{z}(x, y, t)$ is prescribed uniformly in the actuator regions $P_{\mathrm{U}}$ and $P_{\mathrm{L}}$ as

$$
\begin{aligned}
& T_{z}(x, y, t)=g_{\mathrm{U}}(t) \text { in } P_{\mathrm{U}}, \\
& T_{z}(x, y, t)=g_{\mathrm{L}}(t) \text { in } P_{\mathrm{L}}
\end{aligned}
$$

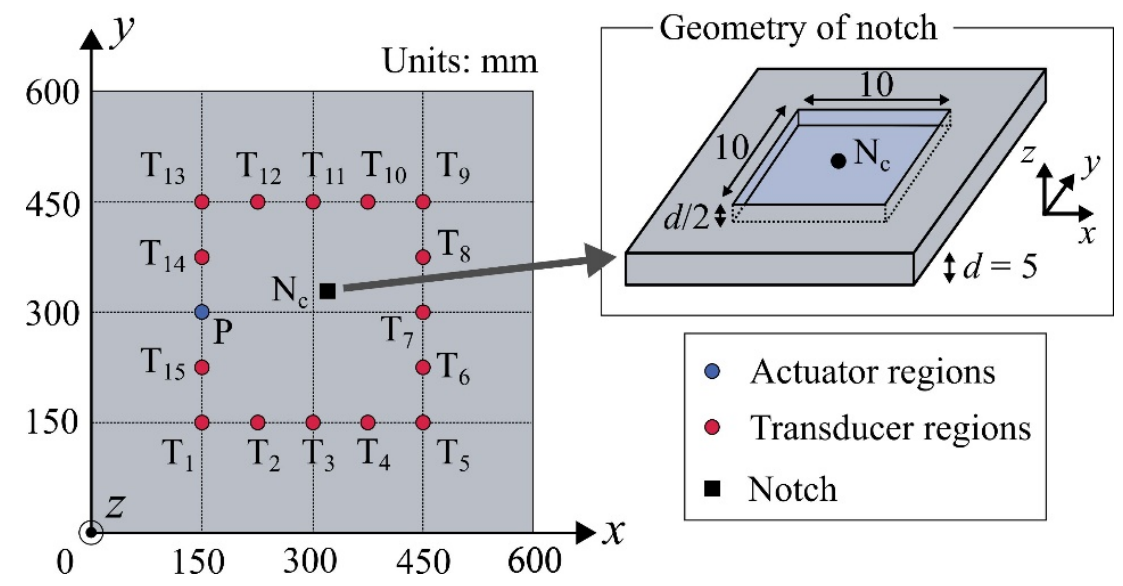

Fig. 2 Numerical model of a square plate with a partial-thickness notch for the calculation of the signals of the forward-propagating S0 and A0 modes. The center coordinates of the dual transducer regions $\mathrm{T}_{1}-\mathrm{T}_{15}$ are given in Table 1 . 
respectively, where $P_{\mathrm{U}}=\left\{(x, y, z) \mid\left(x-x_{\mathrm{P}}\right)^{2}+\left(y-y_{\mathrm{P}}\right)^{2} \leq D^{2} / 4, z=d / 2\right\}$ and $P_{\mathrm{L}}=\{(x, y, z) \mid$ $\left.\left(x-x_{\mathrm{P}}\right)^{2}+\left(y-y_{\mathrm{P}}\right)^{2} \leq D^{2} / 4, z=-d / 2\right\}$ are circular regions, $\left(x_{\mathrm{P}}, y_{\mathrm{P}}\right)$ are their center coordinates, $g_{\mathrm{U}}(t)$ and $g_{\mathrm{L}}(t)$ are input waveforms, and $D$ is the diameter. Hereafter, a pair of the circular regions $P_{\mathrm{U}}$ and $P_{\mathrm{L}}$ is referred to as the dual actuator P. Only the $\mathrm{S} 0$ mode is emitted when $g_{\mathrm{U}}(t)=g_{\mathrm{L}}(t)$, while only the A0 mode is emitted when $g_{\mathrm{U}}(t)=-g_{\mathrm{L}}(t)$.

The emitted Lamb wave propagates in the plate and interacts with the defect. The wave signals are received by multiple dual transducers mounted on the surfaces of the plate. A pair of circular regions $T_{\mathrm{U}, i}=\left\{(x, y, z) \mid\left(x-x_{i}\right)^{2}+\left(y-y_{i}\right)^{2} \leq D^{2} / 4, z=d / 2\right\}$ and $T_{\mathrm{L}, i}=\left\{(x, y, z) \mid\left(x-x_{i}\right)^{2}+\left(y-y_{i}\right)^{2}\right.$ $\left.\leq D^{2} / 4, z=-d / 2\right\}$ is referred to as the dual transducer $\mathrm{T}_{i}\left(i=1,2, \ldots, N_{\mathrm{T}}\right)$, which corresponds to Transducer $i$ introduced in Section 2.1. The electric voltages $e_{i}^{\mathrm{U}}(t)$ and $e_{i}^{\mathrm{L}}(t)$ obtained for $T_{\mathrm{U}, i}$ and $T_{\mathrm{L}, i}$, respectively, are calculated as

$$
\begin{aligned}
& e_{i}^{\mathrm{U}}(t)=k_{\mathrm{s}} v_{i}^{\mathrm{U}}(t), \\
& e_{i}^{\mathrm{L}}(t)=-k_{\mathrm{s}} v_{i}^{\mathrm{L}}(t),
\end{aligned}
$$

where $v_{i}^{\mathrm{U}}(t)$ and $v_{i}^{\mathrm{L}}(t)$ are the spatial averages of the out-of-plane particle velocity in $T_{\mathrm{U}, i}$ and $T_{\mathrm{L}, i}$, respectively, and $k_{\mathrm{S}}$ is the electro-mechanical coefficient of the transducer. As described in Section 2.2, the frequency dependence of the electro-mechanical coefficient is not taken into account in this study. The minus sign appears in the second equation of Eq. (8) to be consistent with the first equation because the outer normals of the circular regions $T_{\mathrm{U}, i}$ and $T_{\mathrm{L}, i}$ have opposite directions. The waveforms of the $\mathrm{S} 0$ and $\mathrm{A} 0$ modes can be calculated as

$$
\begin{aligned}
& e_{\mathrm{S}, i}(t)=\frac{e_{i}^{\mathrm{U}}(t)+e_{i}^{\mathrm{L}}(t)}{2}, \\
& e_{\mathrm{A}, i}(t)=\frac{e_{i}^{\mathrm{U}}(t)-e_{i}^{\mathrm{L}}(t)}{2},
\end{aligned}
$$

due to the symmetry of Lamb modes. Below, the damage localization methods in three different manners are examined including the proposed time reversal method.

\subsection{Numerical TR simulation}

As described in Section 2.2, the signals of the mode-converted scattered Lamb wave from a defect are time-reversed and re-emitted in the proposed method. In this study, two other damage localization methods based on non-mode-converted Lamb waves (Methods I and II) are also examined for comparison.

\subsubsection{Method I (time reversal of both direct and scattered waves)}

In Method I, the signals which correspond to the emitted mode in the forward propagation are time-reversed. Namely, when the S0 mode is emitted in the damaged plate, the time-reversed signal 


$$
e_{i}^{\mathrm{R}}(t)=e_{\mathrm{S}, i}^{\mathrm{Dir}}(T-t)+e_{\mathrm{S}, i}^{\mathrm{Sca}}(T-t),
$$

is used as the input waveform for Transducer $i$, and the S0 mode is re-emitted in the numerical TR simulation. It is noted that the time-reversed signal in Eq. (10) contains not only the scattered wave but also the direct wave from the actuator. The locations of the focusing peaks are identified by calculating $I_{\mathrm{m}}(x, y)$ in Eq. (6) analogously to the proposed method.

\subsubsection{Method II (time reversal of the non-mode-converted scattered wave)}

In Method II, the same mode as the emitted wave in the forward propagation is utilized similarly to Method I. However, the signals of the scattered wave from the defect are extracted by using baseline data. If the scattering at the defect is sufficiently weak, the signals for the undamaged plate correspond to the direct wave from the actuator. When the S0 mode is emitted in the damaged plate, the time-reversed signal

$$
e_{i}^{\mathrm{R}}(t)=e_{\mathrm{S}, i}^{\mathrm{Sca}}(T-t),
$$

is used as the input waveform for Transducer $i$, and the scattered S0 mode is re-emitted in the numerical TR simulation.

\subsection{Numerical model and parameters}

The propagation behavior of Lamb waves in the plates is analyzed by the elastodynamic finite integration technique (EFIT) [35]. The material property of aluminum alloy plates is used in the numerical calculation. The velocities of the longitudinal and transverse waves of the plates are $c_{\mathrm{L}}$ $=6.4 \mathrm{~km} / \mathrm{s}$ and $c_{\mathrm{T}}=3.17 \mathrm{~km} / \mathrm{s}$, respectively, and the mass density is $\rho=2.7 \times 10^{3} \mathrm{~kg} / \mathrm{m}^{3}$. The center coordinates of the dual actuator region, $\mathrm{P}_{\mathrm{c}}$, are $\left(x_{\mathrm{P}}, y_{\mathrm{P}}\right)=(150 \mathrm{~mm}, 300 \mathrm{~mm})$, as shown in Fig. 2. Table 1 shows the center coordinates of the dual transducers $\mathrm{T}_{i}(i=1,2, \ldots, 15)$. The diameter of the transducers is set as $D=10 \mathrm{~mm}$. The dual transducers serve as receivers in the forward propagation, and emitters in the backward propagation. The center coordinates of the square notch, $\mathrm{N}_{\mathrm{c}}$, are set as $\left(x_{\mathrm{N}}, y_{\mathrm{N}}\right)=(320 \mathrm{~mm}, 330 \mathrm{~mm})$.

A 4-cycle tone burst with a given excitation frequency modulated by a Hanning window function is used as the input waveform for the dual actuator $\mathrm{P}$, and the $\mathrm{S} 0$ or $\mathrm{A} 0$ mode is emitted in the damaged plate. Five excitation frequencies $(0.075,0.1,0.15,0.2$, and $0.25 \mathrm{MHz})$ are considered to investigate the effect of frequency on the time reversal focusing characteristics. These excitation frequencies are lower than the cut-off frequencies of the higher-order guided wave modes.

The numerical model of the plate is discretized by cubic cells with the side length of $d / 10=$ $0.5 \mathrm{~mm}$. The size of these cells corresponds to approximately $5 \%$ of the minimum wavelength ( $9.4 \mathrm{~mm}$ for the A0 mode at $0.25 \mathrm{MHz}$ ). The total number of the cells is approximately 14 million. The time increment is set as $34 \mathrm{~ns}$ based on the stability criterion [35]. The algorithm of the EFIT is implemented in $\mathrm{C}++$, and the numerical calculation is run with the aid of parallel computing 
based on OpenMP.

Table 1 Center coordinates of the dual transducers $T_{i}(i=1,2, \ldots, 15)$.

\begin{tabular}{cc|cc}
\hline$i$ & $\left(x_{i}, y_{i}\right)[\mathrm{mm}]$ & $i$ & $\left(x_{i}, y_{i}\right)[\mathrm{mm}]$ \\
\hline 1 & $(150,150)$ & 9 & $(450,450)$ \\
2 & $(225,150)$ & 10 & $(375,450)$ \\
3 & $(300,150)$ & 11 & $(300,450)$ \\
4 & $(375,150)$ & 12 & $(225,450)$ \\
5 & $(450,150)$ & 13 & $(150,450)$ \\
6 & $(450,225)$ & 14 & $(150,375)$ \\
7 & $(450,300)$ & 15 & $(150,225)$ \\
8 & $(450,375)$ & & \\
\hline
\end{tabular}

\section{Spatial focusing of the time-reversed SO and A0 modes in a homogeneous plate}

Prior to the verification of the proposed damage localization method, the spatial focusing characteristics of the time-reversed Lamb waves in a homogeneous plate with no damage are investigated. In this section, the dual actuator is mounted at the location $\mathrm{N}_{c}$, which corresponds to the center location of the notch in the damaged plate of Fig. 2, as shown in Fig. 3.

The S0 mode is excited by the actuator using the input waveform of the center frequency of $0.1 \mathrm{MHz}$, and the signals of the $\mathrm{S} 0$ mode are calculated at the transducers $\mathrm{T}_{i}(i=1,2, \ldots, 15)$ by Eq. (9). Figure 4(a) shows the waveforms of the $S 0$ mode at the four transducers $T_{1}, T_{5}, T_{9}$, and $\mathrm{T}_{13}$. For each waveform, the direct wave from the actuator is located before $100 \mu \mathrm{s}$, and the

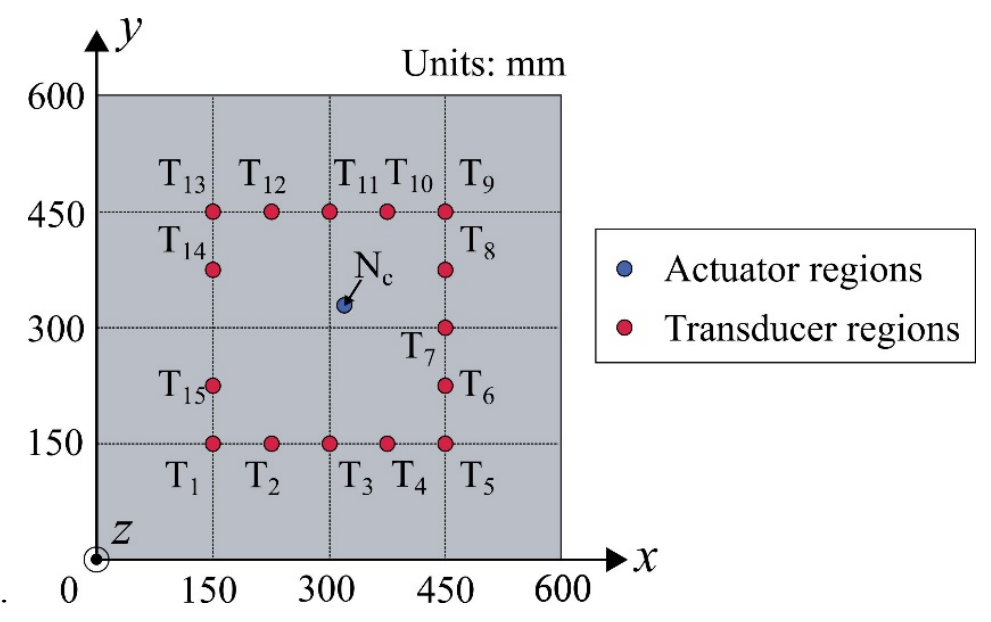

Fig. 3 Numerical model of a homogeneous plate for the investigation of the spatial focusing characteristics of the time-reversed Lamb waves. The location of the dual actuator corresponds to the center location of the notch $\mathrm{N}_{\mathrm{c}}$ shown in Fig. 2. 
reflected waves from the edges of the plate are subsequently detected. The arrival times of the direct wave correspond to the group velocity of the $\mathrm{S} 0$ mode $(5.4 \mathrm{~km} / \mathrm{s})$. The signal of the direct wave is extracted by multiplying each waveform by a tapered rectangular window function. The obtained signal is reversed in $0<t<200 \mu \mathrm{s}$, as shown in Fig. 4(b). This procedure is performed for all transducers, and the time-reversed signals are used as the input waveforms for the backward propagation of the S0 mode. If the effect of the reflection at the plate edges is considered in the time reversal process, the number of virtual sensors increases and the focusing characteristics will be enhanced [2,3]. However, in this section, the reflected waves are eliminated to examine the spatial focusing of the direct waves only.

The propagation of the time-reversed S0 mode is numerically analyzed in $0<t<200 \mu \mathrm{s}$. The calculated focusing index $I_{\mathrm{m}}(x, y)$ is shown in Fig. 5(a), where $I_{\mathrm{m}}(x, y)$ is normalized by the value at the center coordinates of the original wave source $\mathrm{N}_{\mathrm{c}}, I_{\mathrm{m}}\left(x_{\mathrm{N}}, y_{\mathrm{N}}\right)$. It is shown in Fig. 5(a) that the focusing index has a peak at $\mathrm{N}_{\mathrm{c}}$ due to the focusing of the time-reversed $\mathrm{S} 0$ mode at the original wave source. Other peaks which are higher than unity correspond to the wave sources in the numerical TR simulation (i.e. the transducers $\mathrm{T}_{i}$ ). Figure $5(\mathrm{~b})$ shows the focusing index along $y=$
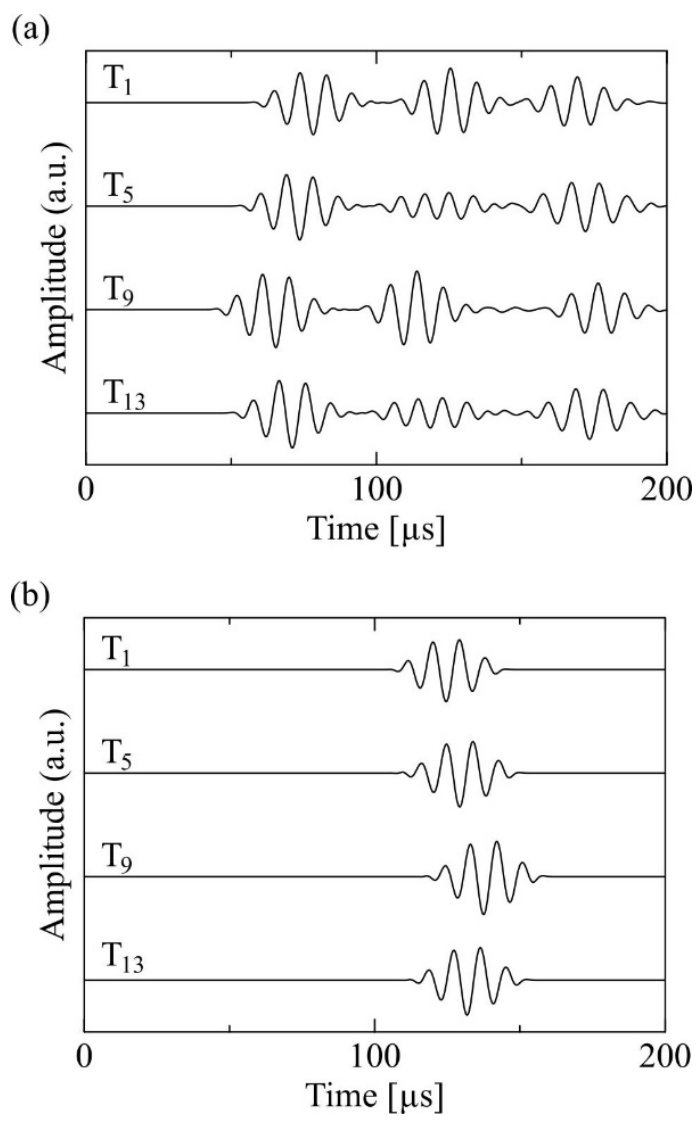

Fig. 4 (a) Waveforms of the $S 0$ mode obtained at the transducers $T_{1}, T_{5}, T_{9}$, and $T_{13}$, and (b) the time-reversed signals of the direct wave, when the $\mathrm{S} 0$ mode is excited in a homogeneous plate using the input waveform of the center frequency of $0.1 \mathrm{MHz}$. 
$y_{\mathrm{N}}$ as a function of the $x$ coordinate, $I_{\mathrm{m}}\left(x, y_{\mathrm{N}}\right)$. In this study, the region around the original wave source location $\mathrm{N}_{\mathrm{c}}$ in which $I_{\mathrm{m}}(x, y)$ becomes more than 0.5 is referred to as the main lobe [30], as indicated in Fig. 5(b). The main lobe is accompanied by two side lobes of the level of

(a)

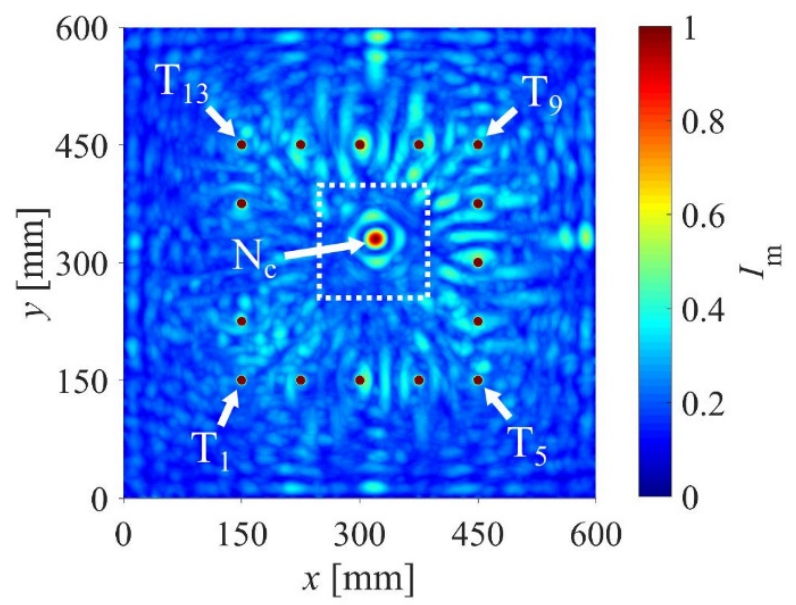

(b)

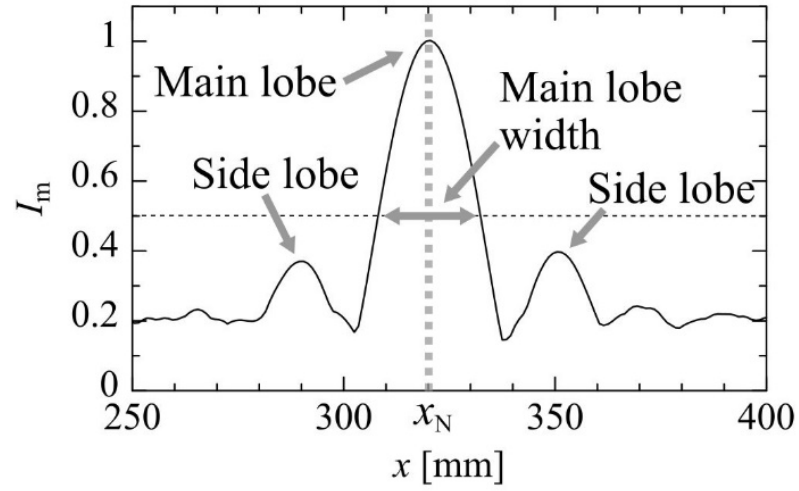

(c)

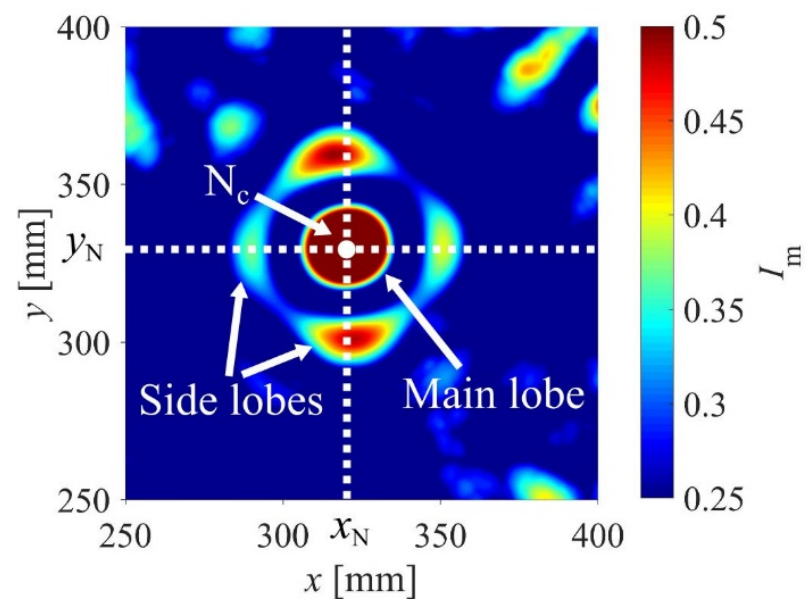

Fig. 5 Focusing indices of the time-reversed S0 mode (a) on the entire plate surface, (b) along $y$ $=y_{\mathrm{N}}$, and (c) around the center of the original wave source $\mathrm{N}_{\mathrm{c}}$. The input waveform of the center frequency of $0.1 \mathrm{MHz}$ is used to emit the S0 mode in a homogeneous plate. The focusing index is normalized by the value at $\mathrm{N}_{\mathrm{c}}$. 
approximately 0.4 , which are located at around $x=290 \mathrm{~mm}$ and $350 \mathrm{~mm}$. The focusing index in the vicinity of $\mathrm{N}_{\mathrm{c}}$ is enlarged in Fig. 5(c), where the color scaling is different from Fig. 5(a). In Fig. 5(c), the main lobe appears in red and has a circular-disk shape with the center at $\mathrm{N}_{\mathrm{c}}$. The relative magnitude of the side lobes is expected to decrease by increasing the number of transducers, or by using time-reversed signals with a longer time duration (i.e. including the reflected waves from the plate edges in the time reversal process) $[2,3]$.

The above numerical simulation is performed for different input frequencies. To examine the spatial focusing characteristics of the time-reversed S0 mode, the width of the main lobe, as indicated in Fig. 5(b), is calculated. This quantity is called spatial resolution in Ref. [30]. The main lobe widths along the $x$ and $y$ directions obtained from the time reversal of the S0 mode are shown in Fig. 6. The horizontal axis represents the frequency at which the amplitude spectrum of the waveform at the transducer $T_{1}$ in the forward propagation shows a maximum value. The peak frequency of the amplitude spectrum does not necessarily coincide with the input frequency because the amplitude response of the excited guided wave mode shows frequency dependence affected by the dimensions of the actuator [38, 39]. In Fig. 6, the main lobe width of the time-reversed S0 (TR S0) mode decreases with increasing frequency. The main lobe widths in the $x$ and $y$ directions have similar values, as indicated by a circular-disk shape of the main lobe in Fig. 5(c). The time reversal focusing of the A0 mode is also simulated when the A0 mode is excited in the homogeneous plate. As shown in Fig. 6, the main lobe width of the A0 mode also takes almost the same values in the $x$ and $y$ directions, and slightly decreases with increasing frequency.

The spatial focusing characteristics of the time-reversed A0 mode were reported by Park [30] using both theoretical and finite element analysis. In Ref. [30], based on the waveform of the A0

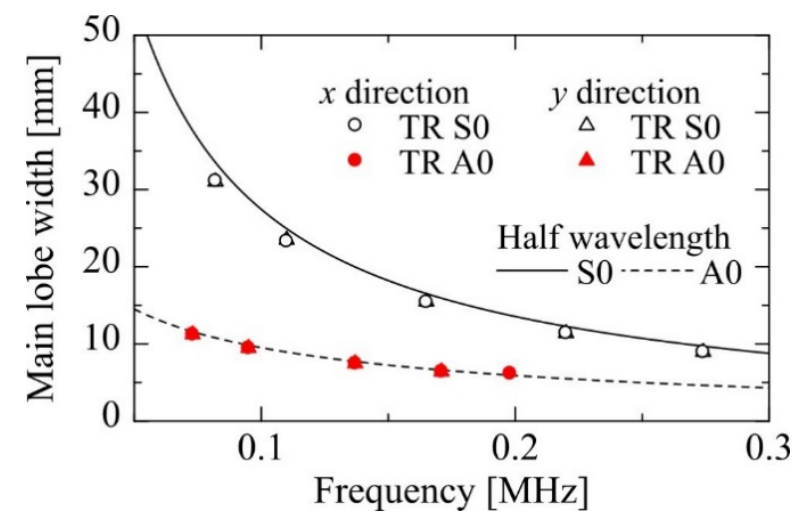

Fig. 6 Frequency dependence of the widths of the main lobes created by the time-reversed (TR) $\mathrm{S} 0$ and $\mathrm{A} 0$ modes when the $\mathrm{S} 0$ and $\mathrm{A} 0$ modes are emitted from a circular wave source in a homogeneous plate, respectively. Solid and dashed lines represent the theoretical values of minimum spatial resolution, which correspond to the half wavelengths of the S0 and A0 modes, respectively. 
mode excited at a point source on a homogeneous plate, the minimum spatial resolution of the time-reversed A0 mode is theoretically predicted as its half wavelength. In Fig. 6, the half wavelengths of the $\mathrm{S} 0$ and $\mathrm{A} 0$ modes are shown together with the main lobe widths. The width of the main lobe created by each time-reversed wave mode is shown to be close to its minimum spatial resolution. The size of the original circular wave source $(D=10 \mathrm{~mm})$ does not seem to affect the variation of the main lobe width.

\section{Time reversal of the scattered S0 and A0 modes at a partial-thickness notch in a plate}

\subsection{Damage localization by the time reversal of the scattered Lamb wave}

The damage localization for the numerical model shown in Fig. 2 is performed by Methods I and II as well as the proposed time reversal method described in Section 2. The S0 mode is excited from the actuator $\mathrm{P}$ by using the input waveform of the center frequency of $0.1 \mathrm{MHz}$. The waveforms of the S0 mode calculated by Eq. (9) are shown in Fig. 7(a) for the four transducers $\mathrm{T}_{1}$, $\mathrm{T}_{5}, \mathrm{~T}_{9}$, and $\mathrm{T}_{13}$. Each waveform contains the direct wave from the actuator, the scattered wave from the partial-thickness notch, and the reflected wave from the edges of the plate. Since the transducers $T_{1}$ and $T_{13}$ are symmetrically located with respect to the actuator $\mathrm{P}$ and the scattering at the notch is weak, the waveform obtained at the transducer $T_{1}$ is analogous to the one at $T_{13}$. This trend is also seen in the waveforms obtained at the transducers $\mathrm{T}_{5}$ and $\mathrm{T}_{9}$.

In Method I, each S0 mode waveform shown in Fig. 7(a) is reversed in a time range of $0<t<$ $250 \mu \mathrm{s}$, and used as the input waveform in the numerical TR simulation of the S0 mode. The obtained focusing index $I_{\mathrm{m}}(x, y)$ is shown in Fig. 8(a), where it is normalized by the value at the center location of the notch $\mathrm{N}_{\mathrm{c}}$. In this study, the focusing peak which the time-reversed wave creates at its original wave source is referred to as a focused spot. In Fig. 8(a), a focused spot appears at the wave source of the direct wave, $\mathrm{P}$, around which four additional peaks can be seen. The four peaks around the focused spot correspond to side lobes described in Section 4. However, no distinct peak can be observed at the location of the notch $\mathrm{N}_{\mathrm{c}}$ because the signals of the scattered wave from the notch are overwhelmed by the direct wave from the actuator. Namely, the focusing peak amplitude by the time reversal of the scattered S0 mode is far lower than the amplitude of the direct wave. Other clear peaks correspond to the wave sources in the numerical TR simulation (i.e. the transducers $\mathrm{T}_{i}$ ). 
(a)

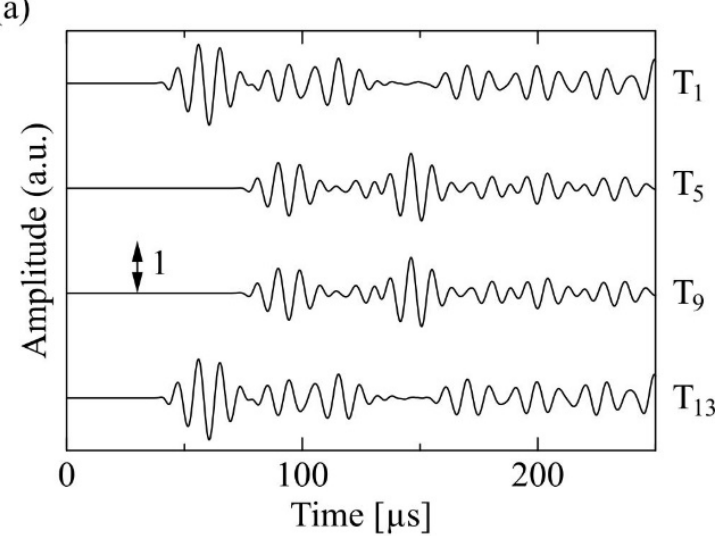

(b)

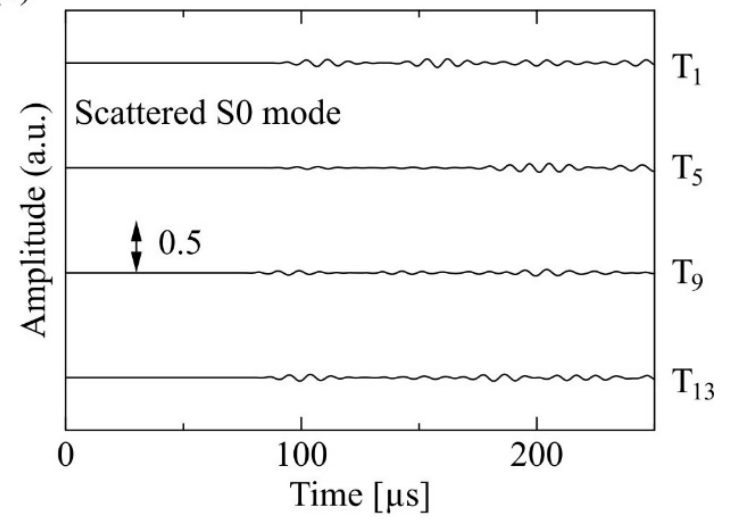

(c)

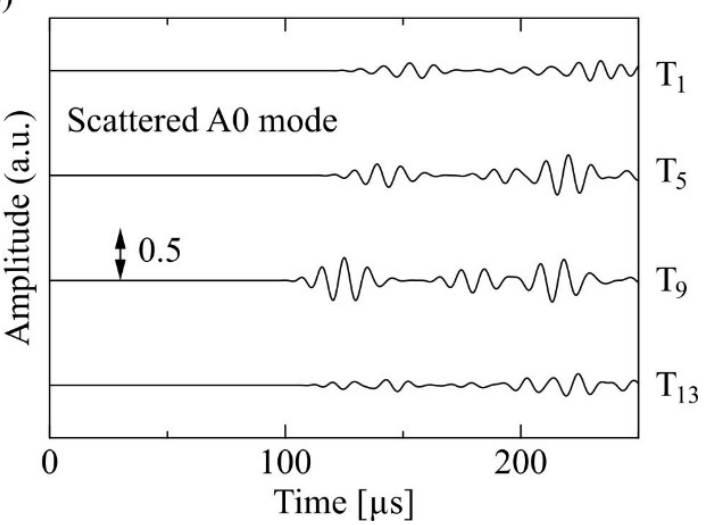

Fig. 7 Waveforms of (a) the S0 mode including the direct wave from the actuator, (b) the scattered S0 mode, and (c) the scattered A0 mode generated by mode conversion at the notch, obtained at different transducers. The waveform of the center frequency of $0.1 \mathrm{MHz}$ is used for the excitation of the S0 mode in the damaged plate. The scaling of the vertical axis in (b) and (c) is different from the one in (a). 
(a) Method I

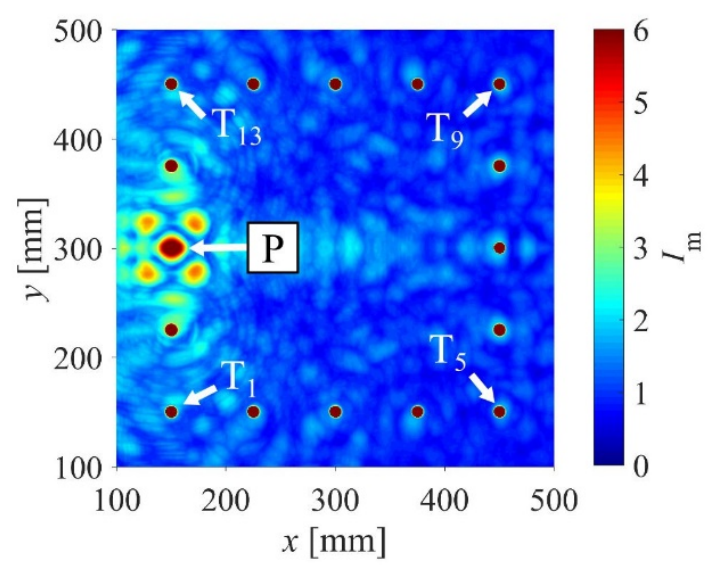

(b) Method II

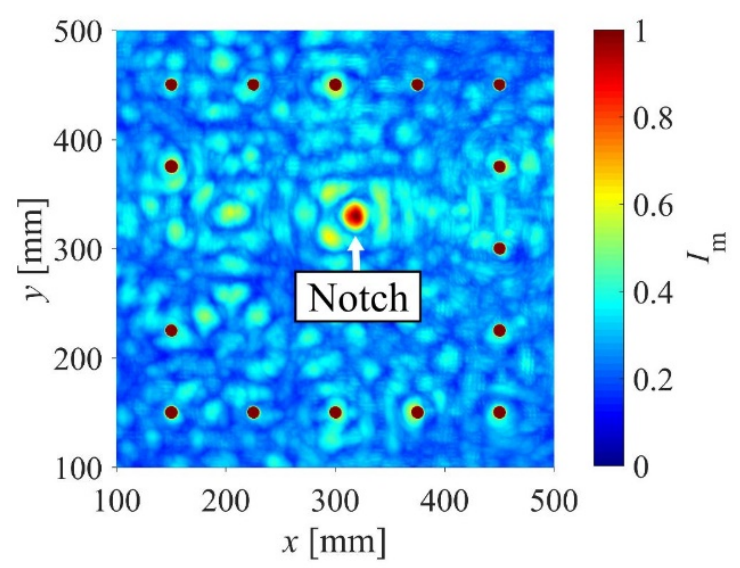

(c) Proposed method

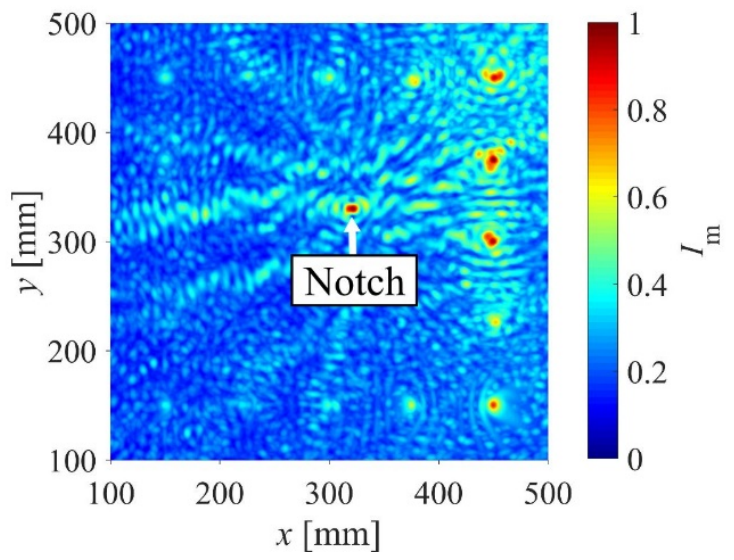

Fig. 8 Focusing indices obtained from the time reversal of (a) the S0 mode including the direct wave from the actuator P (Method I), (b) the scattered S0 mode (Method II), and (c) the scattered A0 mode generated by mode conversion at the notch (the proposed method), when the $\mathrm{S} 0$ mode is excited by using the input waveform of the center frequency of $0.1 \mathrm{MHz}$ in the damaged plate. 
In Method II, the waveforms of the S0 mode calculated for an undamaged plate (i.e. a homogeneous plate with no notch), which correspond to baseline data, are subtracted from the results for the damaged plate in order to eliminate the effect of the direct wave. The waveforms so obtained correspond to the scattered S0 mode from the notch, as shown in Fig. 7(b). The signals of the scattered S0 mode from the notch are time-reversed in $0<t<250 \mu \mathrm{s}$, and then the S0 mode is re-emitted in the numerical TR simulation. The obtained focusing index is shown in Fig. 8(b). A focused spot can be confirmed at the location of the notch because the effect of the direct wave has been successfully excluded. Namely, to create a distinct focused spot at the damage location by the time reversal of the emitted wave mode in the forward propagation, the direct wave from the actuator should be eliminated from the received signals. It is noted that baseline data obtained for an undamaged plate are required in this procedure.

In the proposed time reversal method of Section 2.2, the waveform of the scattered A0 mode from the notch is calculated by Eq. (9) for each transducer, as shown in Fig. 7(c). These waveforms are time-reversed in $0<t<250 \mu \mathrm{s}$, and then the A0 mode is re-emitted in the numerical TR simulation. In Fig. 8(c), the obtained focusing index shows that a focused spot is created at the location of the notch. Since the notch is the only wave source of the A0 mode in the damaged plate, baseline data are not necessary to obtain the waveforms of the scattered A0 mode.

If multiple defects exist in the structure, relatively strong scatterers could be localized using the focusing index defined in Eq. (6). In this case, the detection of the other scatterers would be difficult to achieve because their focused spots are possibly overwhelmed by the time reversal of the scattered wave originating from the strong scatterers. If the DORT (French acronym for the decomposition of time reversal operator) [5, 40,41], which is widely utilized for media with multiple scatterers, is incorporated into the proposed method, the selective focusing of the mode-converted Lamb wave on each defect would become possible. However, this topic is left for a future study.

The damage localization by the three methods is also carried out when the A0 mode is excited in the damaged plate by using the same input waveform as the S0 mode. The focusing indices obtained by Methods I, II, and the proposed method are shown in Figs. 9(a)-(c), respectively. In Method I, a focused spot is not created at the location of the notch but at the original actuator location $\mathrm{P}$, as shown in Fig. 9(a). This trend is analogous to the focusing result of the S0 mode by Method I shown in Fig. 8(a). In Method II (Fig. 9b) and the proposed method (Fig. $9 \mathrm{c}$ ), focused spots can be seen in the vicinity of the notch location. Hereafter, only the spatial focusing characteristics by Method II and the proposed method are examined. 
(a) Method I

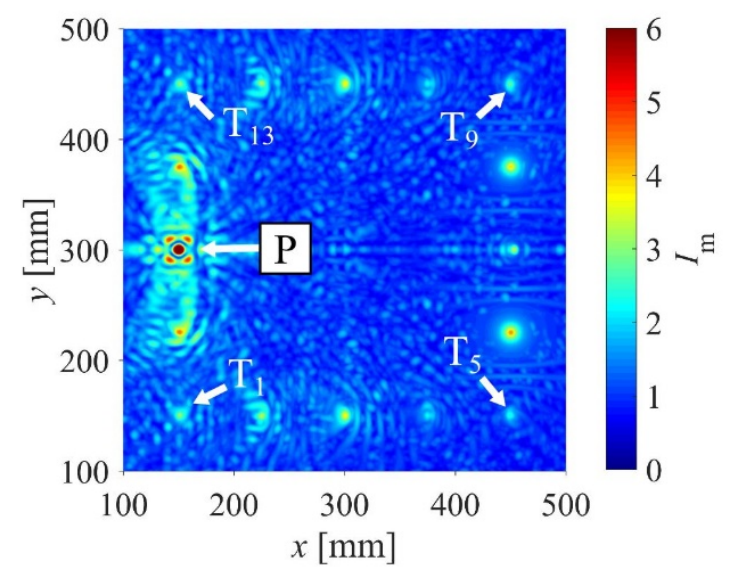

(b) Method II

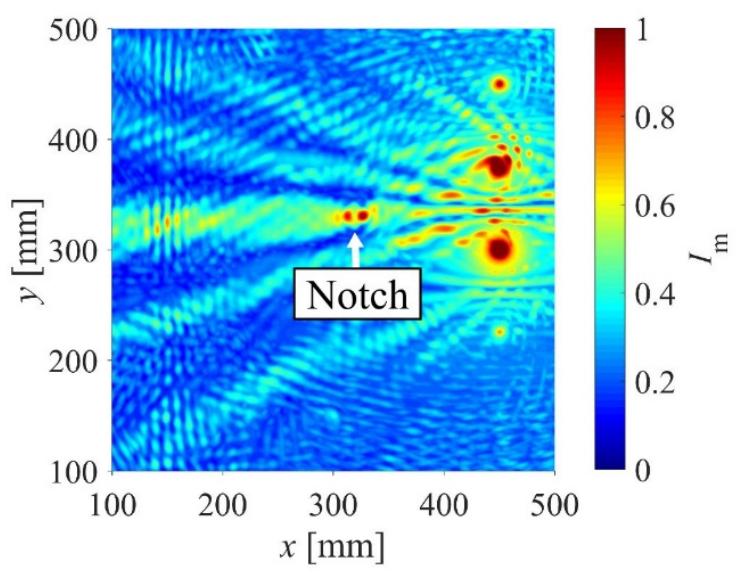

(c) Proposed method

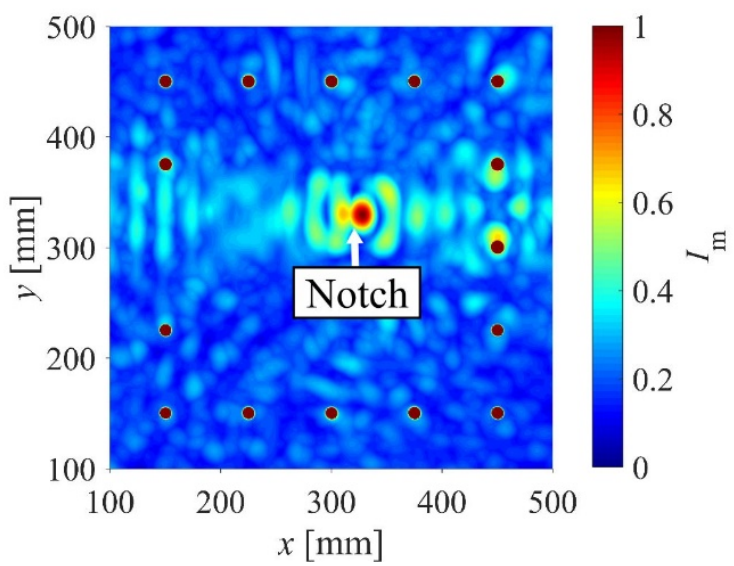

Fig. 9 Focusing indices obtained from the time reversal of (a) the A0 mode including the direct wave from the actuator P (Method I), (b) the scattered A0 mode (Method II), and (c) the scattered S0 mode generated by mode conversion at the notch (the proposed method), when the $\mathrm{A} 0$ mode is excited by using the input waveform of the center frequency of $0.1 \mathrm{MHz}$ in the damaged plate. 
5.2 Comparison of the spatial focusing characteristics by the time reversal of mode-converted and non-mode-converted Lamb waves

\subsubsection{Focusing indices in the vicinity of the notch location}

The spatial focusing characteristics of the time-reversed scattered Lamb wave are investigated for Method II and the proposed method. The focusing indices $I_{\mathrm{m}}(x, y)$ shown in Figs. 8(b), (c), Figs. 9(b), and (c) are enlarged in the vicinity of the notch location in Figs. 10(a)-(d), respectively. In each figure, the focusing index is normalized by its maximum value. Open black squares represent the location and dimensions of the notch. When the S0 mode is emitted in the damaged plate, a focused spot can be seen at the center location of the notch by both Method II and the proposed method, as shown in Figs. 10(a) and (b). The focused spot is accompanied by the peaks of the magnitude of approximately 0.6 around the notch location, which are called artifacts in this

(a) Method II (TR S0)

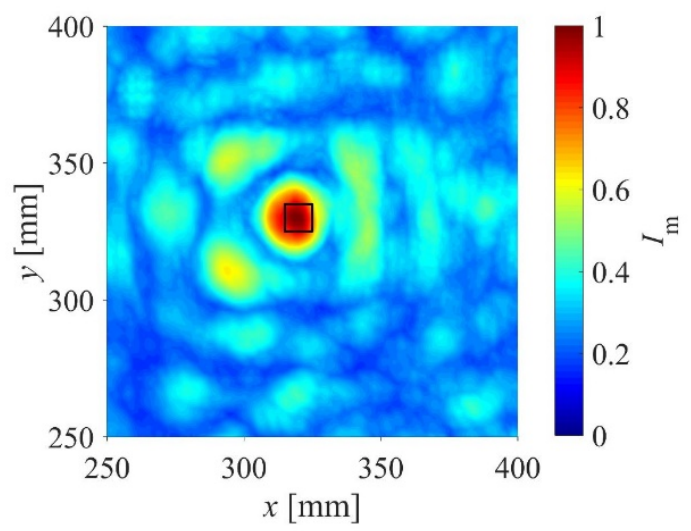

(c) Method II (TR A0)

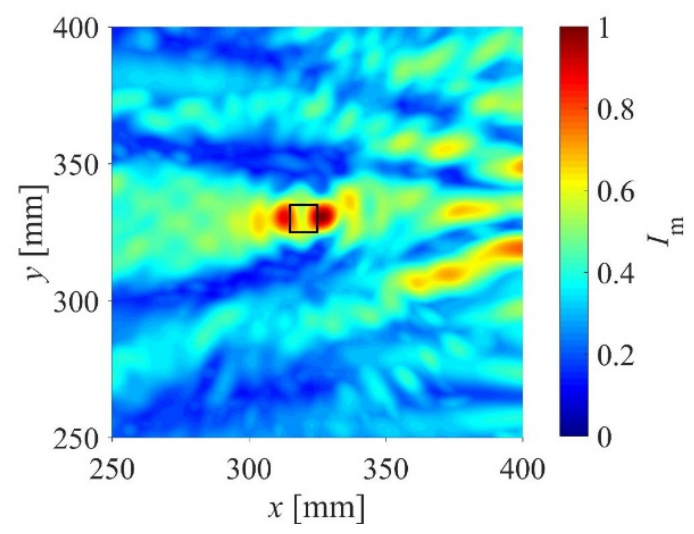

(b) Proposed method (TR A0)

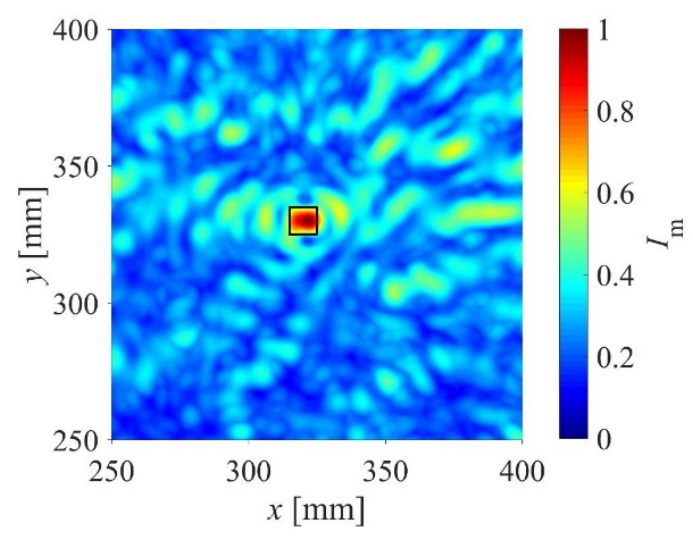

(d) Proposed method (TR S0)

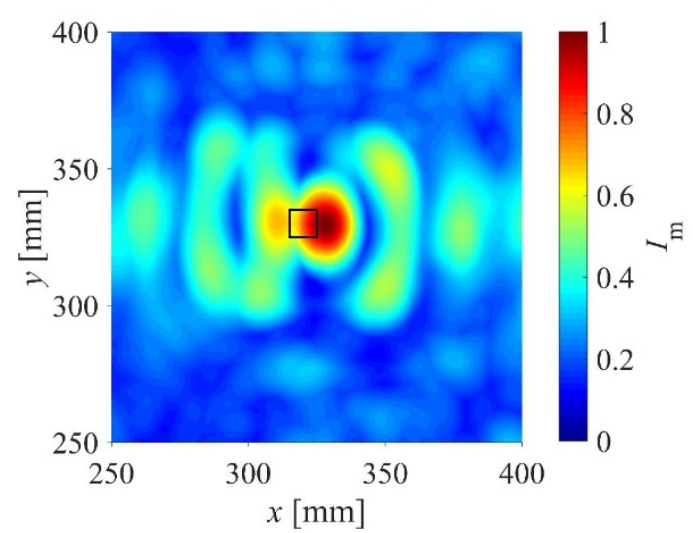

Fig. 10 Enlarged views of the focusing indices obtained by (a) Method II and (b) the proposed method when the S0 mode is excited in the damaged plate, and by (c) Method II and (d) the proposed method when the A0 mode is excited in the damaged plate using the input waveform of the center frequency of $0.1 \mathrm{MHz}$. Open black squares represent the location and dimensions of the notch. 
study. When the A0 mode is emitted in the damaged plate, on the other hand, focused spots are generated on the left and right edges of the notch in Figs. 10(c) and (d). The double peaks indicate that scattering occurred at two step-like discontinuities of the notch. In the following sections, (1) artifacts generated by the time reversal focusing of the scattered wave and (2) the size of the focused spots at the location of the notch are separately discussed.

\subsubsection{Artifacts}

As shown in Fig. 10(c), when the A0 mode is emitted in the damaged plate, the focusing index of the time-reversed A0 mode obtained by Method II is widely distributed compared to the other three cases in Figs. 10 (a), (b), and (d). The magnitude of the focusing index increases not only at the edges of the notch but also at other locations, especially along $y=y_{\mathrm{N}}=330 \mathrm{~mm}$, where $y_{\mathrm{N}}$ is the $y$ coordinate of the center location of the notch. These artifacts could deteriorate the relative focusing amplitude at the notch location.

To examine the reason for non-negligible artifacts in Fig. 10(c), the maximum amplitudes of the scattered waveform obtained at each transducer are shown in Figs. 11(a) and (b) when the S0

(a)

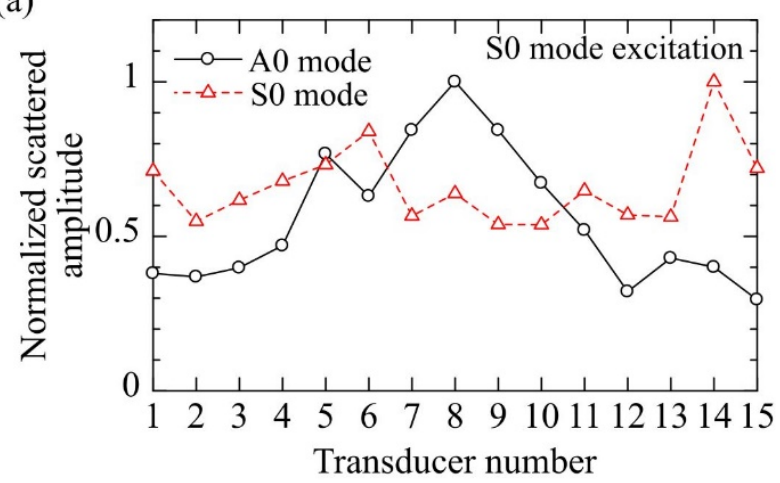

(b)

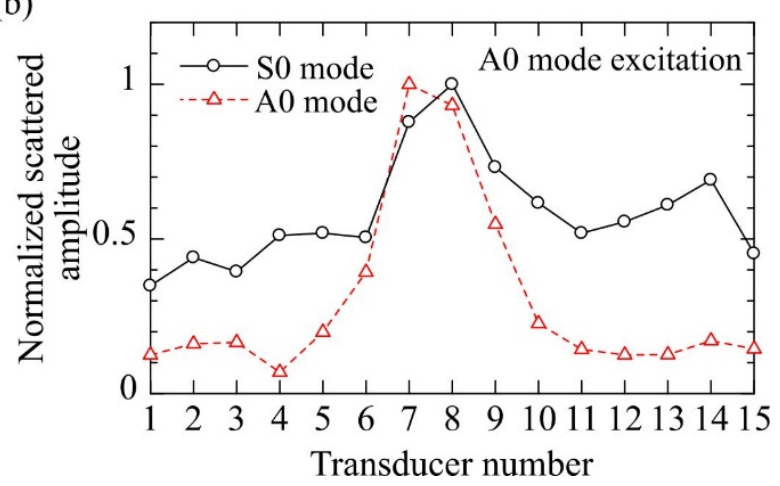

Fig. 11 Maximum amplitudes of the scattered $\mathrm{S} 0$ and $\mathrm{A} 0$ modes obtained at the transducers $\mathrm{T}_{i}(i$ $=1,2, \ldots, 15$ ) when the (a) S0 and (b) A0 modes are excited in the damaged plate using the input waveform of the center frequency of $0.1 \mathrm{MHz}$. The vertical axis is normalized by the maximum value for each scattered wave mode. 
and A0 modes are emitted in the damaged plate, respectively. The scattered amplitude is normalized by the maximum value for each scattered wave mode. In Fig. 11(a), when the S0 mode is emitted, the scattered amplitudes of the S0 and A0 modes are larger than 0.3 at all transducers. When the A0 mode is emitted, the amplitude of the scattered S0 mode shows a similar trend in Fig. 11(b). However, the amplitude of the scattered A0 mode is found to be concentrated in the two transducers $\mathrm{T}_{7}$ and $\mathrm{T}_{8}$ in Fig. 11(b). This fact implies the decrease of the number of virtual sensors for the detection of the scattered A0 mode, which could lead to the imperfect focusing of the time-reversed A0 mode. Artifacts by the time reversal focusing of the A0 mode are distributed mainly along $y=y_{\mathrm{N}}$ in Fig. 10(c) because the center coordinates of the two transducers $\mathrm{T}_{7}$ and $\mathrm{T}_{8}$ satisfy $y_{7}<y_{\mathrm{N}}<y_{8}$, where the $y$ coordinates $y_{7}$ and $y_{8}$ are given in Table 1. Namely, the arrangement of transducers and the choice of the Lamb wave mode have a significant effect on the artifacts. Since the distribution of the scattered A0 mode amplitude is relatively wide in Fig. 11(a), the focusing index of the time-reversed A0 mode by the proposed
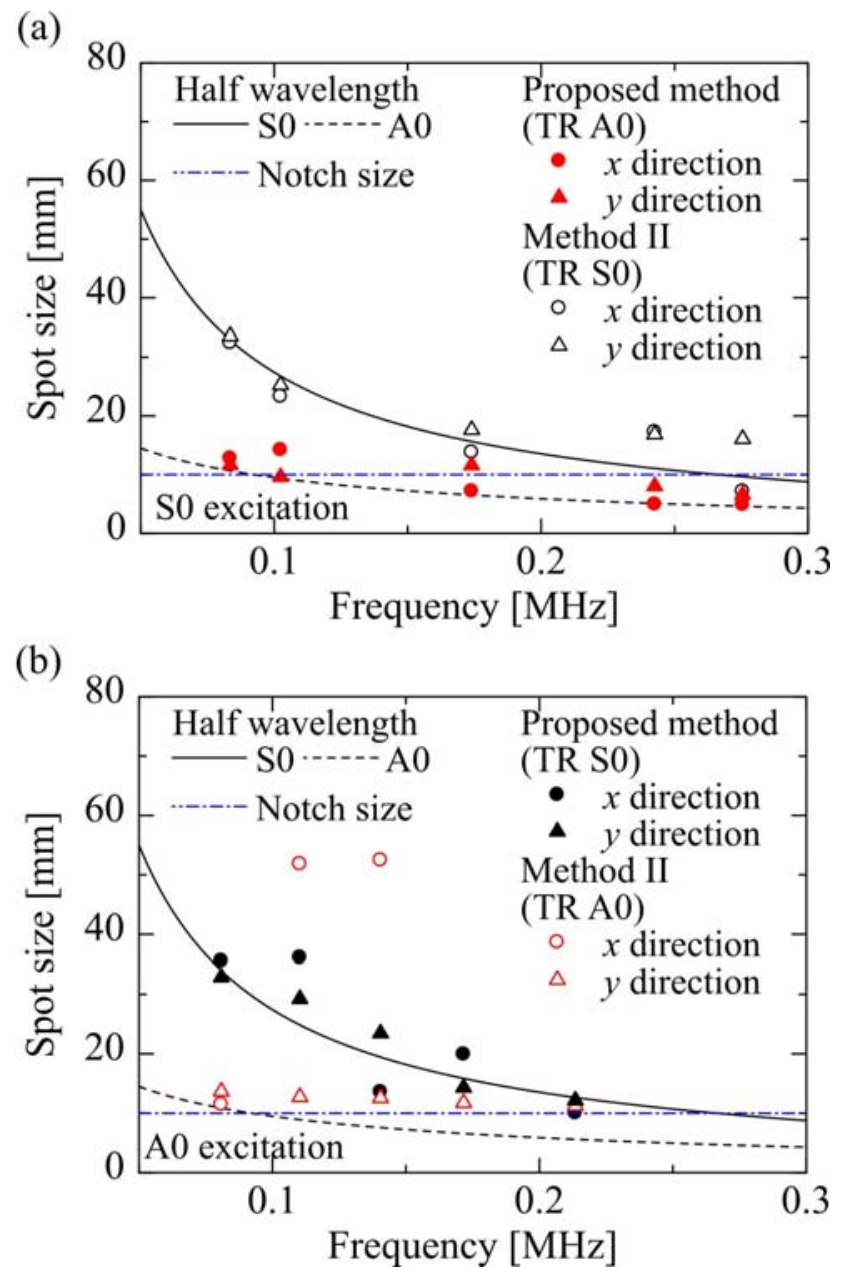

Fig. 12 Sizes of the focused spots in the $x$ and $y$ directions obtained by the proposed method and Method II when the (a) S0 and (b) A0 modes are emitted in the damaged plate. The half wavelengths of the S0 and A0 modes and the notch size (side length $10 \mathrm{~mm}$ ) are shown together. 
method does not show distinct artifacts in Fig. 10(b), compared to the result by Method II in Fig. $10(\mathrm{c})$.

\subsubsection{Size of the focused spots at the notch location}

The time reversal focusing by the proposed method and Method II is numerically analyzed for different input frequencies. The sizes of the focused spots at the location of the notch are calculated in a similar manner to the width of the main lobe in Section 4. When multiple focused spots appear at the left and right edges of the notch as shown in Figs. 10(c) and (d), the one with the higher focusing index is chosen to calculate the spot size. For the excitation of the S0 and A0 modes in the damaged plate, the obtained spot sizes in the $x$ and $y$ directions are shown against the frequency in Figs. 12(a) and (b), respectively. In both figures, the spot sizes show direction dependence, which has not been observed in the focusing characteristics at a circular wave source in Section 4. While the source size does not seem to affect the main lobe width in Fig. 6, the direction dependence in Fig. 12 implies that the geometry and size of the notch influence the focused spot size. The side length of the square notch $(10 \mathrm{~mm})$ and the half wavelengths of the S0 and A0 modes are shown together in Fig. 12.

When the S0 mode is emitted in the damaged plate, the direction dependence of the spot size is not significant, as shown in Fig. 12(a). The spot size of the time-reversed S0 mode by Method II tends to decrease with increasing frequency but is still larger than the notch size. The spot size of the time-reversed A0 mode by the proposed method slightly decreases with the frequency and is close to the notch size, compared to the result of the S0 mode. The spot size of the time-reversed scattered wave is associated with not only its half wavelength but also the notch size.

When the A0 mode is emitted in the forward propagation, the time-reversed A0 mode by Method II also shows the focused spot size close to the notch size in the $y$ direction, as shown in Fig. 12(b). However, the spot size in the $x$ direction by Method II becomes far larger than the half wavelength of the $\mathrm{A} 0$ mode and the notch size above $0.1 \mathrm{MHz}$ (out of range in the vertical axis at around $0.2 \mathrm{MHz}$ in Fig. 12b). This result appears to be due to the imperfect focusing suggested in Section 5.2.2, which makes the focused region broad particularly in the $x$ direction. On the other hand, the spot sizes of the time-reversed S0 mode in both $x$ and $y$ directions by the proposed method tend to decrease with increasing frequency and become almost equal to the notch size at around 0.2 MHz. Even when the A0 mode is emitted in the forward propagation, the size of the focused spot by the proposed method is found to be affected by the defect size and the half wavelength of the time-reversed wave.

In previous studies $[42,43]$, the algorithm for the quantitative damage imaging based on time reversal was proposed, and its advantage was discussed by comparing to modified diffraction tomography and beamforming method. It was demonstrated that the size of modeled laminar damage can be successfully estimated by these algorithms when the Born approximation is satisfied. The diffraction tomography algorithm was extended to the damage imaging utilizing the mode-converted Lamb wave. In the proposed method of the present study, on the other hand, the 
localization of a defect can be achieved but its quantitative estimation seems to be not straight-forward. Nevertheless, since the focusing index $I_{\mathrm{m}}(x, y)$ used in the proposed method is simple, the implementation is easy compared to the aforementioned algorithms, which require relatively complicated procedures using Green functions. The modification of the data processing in the proposed method could improve the quality of the damage estimation, but this topic remains as future work.

\section{Localization of a notch-type defect in a plate with a through-thickness hole}

In this section, the proposed method is applied to a plate with a notch-type defect adjacent to an a priori known through-thickness hole. As shown in Fig. 13, the numerical model of a plate with a square through hole of side length $40 \mathrm{~mm}$ is considered. The dimensions of the plate are the same as the numerical model in Section $3(600 \mathrm{~mm} \times 600 \mathrm{~mm} \times 5 \mathrm{~mm})$. The through hole is located at the center of the plate and has a square partial-thickness notch (side lengths $10 \mathrm{~mm}$ in the $x$ and $y$ directions, and depth $2.5 \mathrm{~mm}$ in the $z$ direction) at the upper edge of the hole. The localization of the partial-thickness notch is carried out by the proposed method. The configuration of the actuator and the transducers is the same as the previous numerical model.

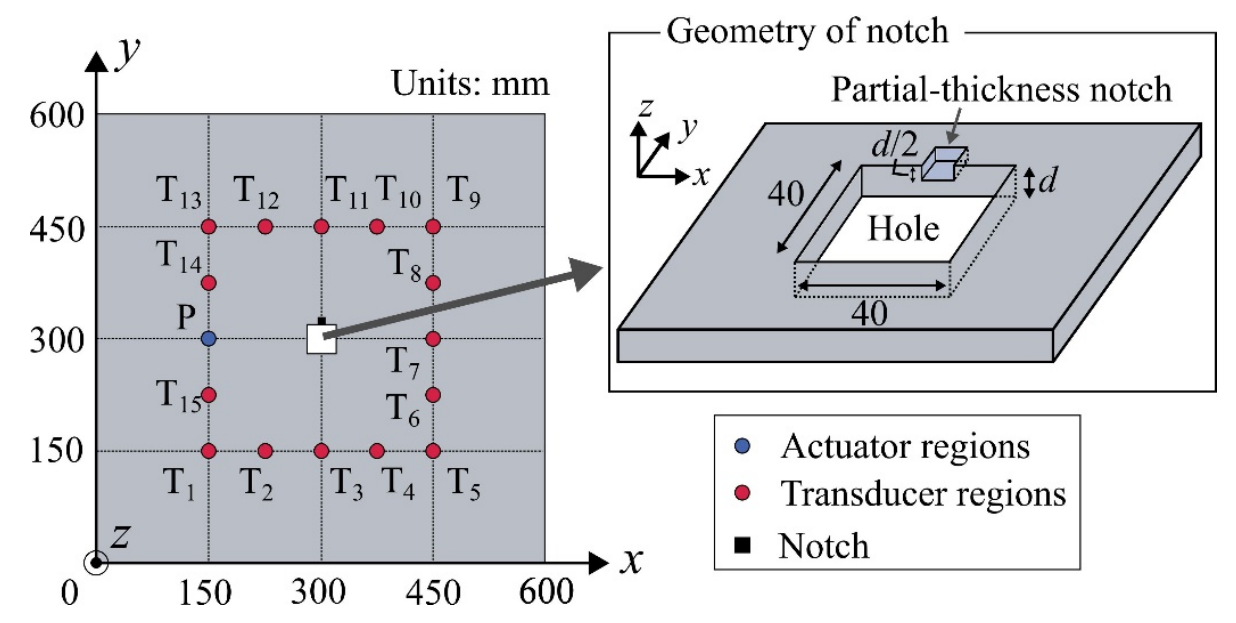

Fig. 13 Numerical model of a plate with a square through-thickness hole for the damage localization by the proposed method. The through hole has a square partial-thickness notch at its upper edge.

The S0 mode is excited from the actuator $\mathrm{P}$ by using the input waveform of the center frequency of $0.1 \mathrm{MHz}$, and then the waveforms of the A0 mode are calculated at the transducers $\mathrm{T}_{1}-\mathrm{T}_{15}$ in a time range of $0<t<250 \mu$ s by Eq. (9). The obtained waveforms correspond to the scattered wave signals from the partial-thickness notch because the mode conversion between symmetric and antisymmetric Lamb modes does not occur except at the notch.

The numerical TR simulation of the scattered A0 mode is performed for a plate which has the 
through-thickness hole with no notch. The obtained focusing index around the hole is shown in Fig. 14, together with an open black square representing the location and dimensions of the notch. This result implies that the scattered A0 mode generated from the edges of the notch (i.e. the step-like discontinuities) is successfully focused at its original wave sources by the proposed method. While the time-reversed A0 mode by the proposed method shows a focused spot at the center location of the notch for the plate with no hole in Fig. 10(b), the magnitude of the focusing index in Fig. 14 is found to increase along the edges of the notch. A possible explanation for this difference is that the time-reversed A0 mode for the plate with the hole converges at the notch location via different propagation paths which permit the multiple reflection and scattering at the edges of the hole and the notch. This effect is likely to increase the virtual number of transducers and enhance the focusing amplitude. The focusing index also increases at the upper edge of the square hole because the reflection of the scattered A0 mode occurs at the hole edge.

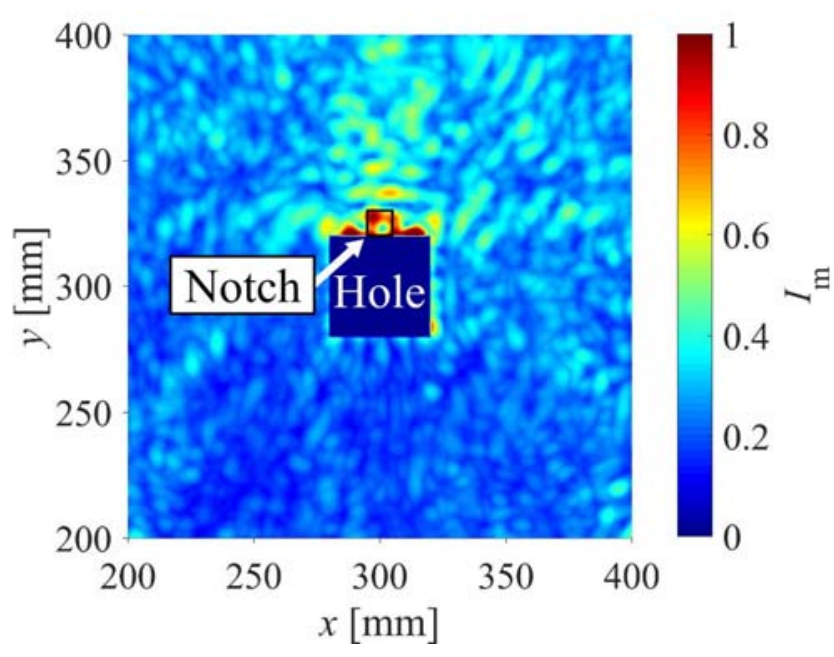

Fig. 14 Focusing index obtained from the time reversal of the scattered A0 mode by the proposed method when the $\mathrm{S} 0$ mode is excited in the damaged plate using the input waveform of the center frequency of $0.1 \mathrm{MHz}$. An open black square represents the location and dimensions of the notch.

\section{Conclusion}

A damage localization method by the time reversal of the mode-converted Lamb wave has been proposed for plates with a non-symmetric defect in the thickness direction. In the proposed method, the signals of the mode-converted Lamb wave generated at the defect are measured at multiple transducers, and the localization of the damage is performed by the numerical time-reversed (TR) simulation based on the measured signals. To selectively emit and receive the S0 and A0 modes below the cut-off frequencies of the higher-order guided modes, dual transducers are attached symmetrically on the upper and lower surfaces of the plate. In this study, the elastodynamic finite integration technique (EFIT) has been used to simulate the signals of the 
Lamb waves in a plate with a notch-type defect instead of the measurement. When the Lamb mode which corresponds to the emitted wave mode in the damaged plate is time-reversed, focusing peaks do not appear at the location of the notch without the extraction of the scattered wave signals using baseline data for the undamaged plate. However, the proposed method, namely, the time reversal of the mode-converted scattered wave, enables the focusing at the damage location without the baseline data. It has been shown that the proposed method reduces the magnitude of artifacts and creates focused spots at the notch location, whose sizes are associated with the size of the notch and the half wavelength of the time-reversed wave mode. Furthermore, the localization of a partial-thickness notch adjacent to a square through-thickness hole has been carried out. The proposed method has shown potential to achieve the localization of damage in a relatively complex plate structure, such as a plate with through-thickness holes. In future studies, experimental verification is necessary to discuss the applicability of the proposed method to the damage localization for real plate structures.

\section{Acknowledgement}

This work has been supported by JSPS KAKENHI Grant Number 26-2190.

\section{Reference}

[1] M. Fink, Time reversal of ultrasonic fields-Part I: Basic principles, IEEE Trans. Ultrason. Ferroelectr. Freq. Control 39 (1992) 555-566.

[2] C. Draeger, M. Fink, One-channel time reversal of elastic waves in a chaotic 2D-silicon cavity, Phys. Rev. Lett. 79 (1997) 407-410.

[3] P. Roux, M. Fink, Time reversal in a waveguide: Study of the temporal and spatial focusing, J. Acoust. Soc. Am. 107 (2000) 2418-2429.

[4] B. V. Damme, K. Van Den Abeele, Y. Li, O. Mater, Time reversed acoustics techniques for elastic imaging in reverberant and nonreverberant media: An experimental study of the chaotic cavity transducer concept, J. Apply. Phys. 109 (2011) 104910.

[5] L. Ambrozinski, T. Stepinski, P. Packo, T. Uhl, Self-focusing Lamb waves based on the decomposition of the time-reversal operator using time-frequency representation, Mech. Syst. Signal Process. 27 (2012) 337-349.

[6] P.-Y. Le Bas, M. C. Remillieux, L. Pieczonka, J. A. Ten Cate, B. E. Anderson, T. J. Ulrich, Damage imaging in a laminated composite plate using an air-coupled time reversal mirror, Appl. Phys. Lett. 107 (2015) 184102.

[7] T. Leutenegger, J. Dual, Detection of defects in cylindrical structures using a time reverse method and a finite-difference approach, Ultrasonics 40 (2002) 721-725.

[8] R. K. Ing, N. Quieffin, S. Catheline, M. Fink, In solid localization of finger impacts using acoustic time-reversal process, Appl. Phys. Lett. 87 (2005) 204104.

[9] T. J. Ulrich, A. M. Sutin, R. A. Guyer, P. A. Johnson, Time reversal and non-linear elastic wave spectroscopy (TR NEWS) techniques, Int. J. Non-linear Mech. 43 (2008) 209-216. 
[10] F. Ciampa, M. Meo, Impact detection in anisotropic materials using a time reversal approach, Struct. Health Monitor. 11 (2011) 43-49.

[11] F. Ciampa, M. Meo, Nonlinear elastic imaging using reciprocal time reversal and third order symmetry analysis, J. Acoust. Soc. Am. 131 (2012) 4316-4323.

[12] X. Guo, D. Zhang, J. Zhang, Detection of fatigue-induced micro-cracks in a pipe by using time-reversed nonlinear guided waves: A three-dimensional model study, Ultrasonics 52 (2012) 912-919.

[13] K. Nakahata, K. Kimoto, Reconstruction of flaws in heterogeneous media using image-based FIT and time reversal approach, J. Solid. Mech. Mater. Eng. 6 (2012) 771-781.

[14] R. Ernst, J. Dual, Acoustic emission localization in beams based on time reversed dispersion, Ultrasonics 54 (2014) 1522-1533.

[15] R. Ernst, J. Dual, Quantitative guided wave testing by applying the time reversal principle on dispersive waves in beams, Wave Motion 58 (2015) 259-280.

[16] R. Seidl, E. Rank, Iterative time reversal based flaw identification, Comput. Math. with Appl. 72 (2016) 879-892.

[17] P. D. Wilcox, A rapid signal processing technique to remove the effect of dispersion from guided wave signals, IEEE Trans. Ultrason. Ferroelectr. Freq. Control 50 (2003) 419-427.

[18] D. Alleyne, P. Cawley, A two-dimensional Fourier transform method for the measurement of propagating multimode signals, J. Acoust. Soc. Am. 89 (1991) 1159-1168.

[19] K. Kishimoto, H. Inoue, M. Hamada, T. Shibuya, Time frequency analysis of dispersive waves by means of wavelet transform, J. Appl. Mech. 62 (1995) 841-846.

[20] J. Jiao, C. He, B. Wu, R. Fei, X. Wang, Application of wavelet transform on modal acoustic emission source location in thin plates with one sensor, Int. J. Press. Vessel. Pip. 81 (2004) 427-431.

[21] S. M. H. Hosseini, S. Duczek, U. Gabbert, Damage localization in plates using mode conversion characteristics of ultrasonic guided waves, J. Nondestruct. Eval. 33 (2014) $152-165$.

[22] R. K. Ing, M. Fink, Self-focusing and time recompression of Lamb waves using a time reversal mirror, Ultrasonics 36 (1998) 179-186.

[23] H. W. Park, H. Sohn, K. H. Law, C. R. Farrar, Time reversal active sensing for health monitoring of a composite plate, J. Sound Vib. 302 (2007) 50-66.

[24] B. Xu, V. Giurgiutiu, Single mode tuning effects on Lamb wave time reversal with piezoelectric wafer active sensors for structural health monitoring, J. Nondestruct. Eval. 26 (2007) 123-134.

[25] R. Gangadharan, C. R. L. Murthy, S. Gopalakrishnan, M. R. Bhat, Time reversal technique for health monitoring of metallic structure using Lamb waves, Ultrasonics 49 (2009) 696-705.

[26] H. W. Park, S. B. Kim, H. Sohn, Understanding a time reversal process in Lamb wave 
propagation, Wave Motion 46 (2009) 451-467.

[27] Y. Jun, U. Lee, Computer-aided hybrid time reversal process for structural health monitoring, J. Mech. Sci. Tech. 26 (2012) 52-61.

[28] Z. Liu, X. Zhong, T. Dong, C. He, B. Wu, Delamination detection in composite plates by synthesizing time-reversed Lamb waves and a modified damage imaging algorithm based on RAPID, Struct. Contr. Health Monitor. 24 (2017) e1919.

[29] I. Núnez, C. Negreira, Efficiency parameters in time reversal acoustics: Applications to dispersive media and multimode wave propagation, J. Acoust. Soc. Am. 117 (2005) 1202-1209.

[30] H. W. Park, Numerical simulation and investigation of the spatial focusing of time reversal A0 Lamb mode using circular piezoelectric transducers collocated on a rectangular plate, J. Sound Vib. 332 (2013) 2672-2687.

[31] H. Zophoniasson, C. Hudin, C. Bolzmacher, M. Hafez, Dependence of time reversal of acoustic waves in plates on mean frequency and plate's characteristics, Microsyst. Technol. 22 (2016) 1791-1798.

[32] C. H. Wang, J. T. Rose, F.-K. Chang, A synthetic time-reversal imaging method for structural health monitoring, Smart Mater. Struct. 13 (2004) 415-423.

[33] L. Yu, C. A. C. Leckey, Lamb wave-based quantitative crack detection using a focusing array algorithm, J. Intell. Mater. Syst. Struct. 24 (2013) 1138-1152.

[34] Z. Qiu, L. Qiu, J. Yuan, G. Lu, On research of a phase synthesis time reversal focusing method for damage imaging of complex composite structures, J. Intell. Mater. Syst. Struct. 24 (2013) 209-225.

[35] P. Fellinger, R. Marklein, K. J. Langenberg, S. Klaholz, Numerical modeling of elastic wave propagation and scattering with EFIT-elastodynamic finite integration technique, Wave Motion 21 (1995) 47-66.

[36] C. Yang, L. Ye, Z. Su, M. Bannister, Some aspects of numerical simulation for Lamb wave propagation in composite laminates, Comp. Struct. 75 (2006) 267-275.

[37] Y. Lu, L. Ye, Z. Su, C. Yang, Quantitative assessment of through-thickness crack size based on Lamb wave scattering in aluminum plates, NDT\&E Int. 41 (2008) 58-68.

[38] A. Raghavan, C. E. S. Cesnik, Finite-dimensional piezoelectric transducer modeling for guided wave based structural health monitoring, Smart Mater. Struct. 14 (2005) 1448-1461.

[39] V. Giurgiutiu, Tuned Lamb wave excitation and detection with piezoelectric wafer active sensors for structural health monitoring, J. Intell. Mater. Syst. Struct. 16 (2005) 291-305.

[40] C. Prada, S. Manneville, D. Spoliansky, M. Fink, Decomposition of the time reversal operator: Detection and selective focusing on two scatterers, J. Acoust. Soc. Am. 99 (1996) 2067-2076.

[41] J. He, F.-G. Yuan, Lamb wave-based subwavelength damage imaging using the DORT-MUSIC technique in metallic plates, Struct. Heal. Monit. 15 (2016) 65-80. 
[42] L. R. F. Rose, E. Chan, C. H. Wang, A comparison and extensions of algorithms for quantitative imaging of laminar damage in plates. I. Point spread functions and near field imaging, Wave Motion 58 (2015) 222-243.

[43] E. Chan, L. R. F. Rose, C. H. Wang, A comparison and extensions of algorithms for quantitative imaging of laminar damage in plates. II. Non-monopole scattering and noise tolerance, Wave Motion 66 (2016) 220-237. 